\title{
CRISISLAB - Shelfware, Wetware and Sweatware: Realization, Actualization and Simulation Weapons of First Resort
}

\author{
$\underline{\text { J. Truscott }}^{\text {a }}$ \\ ${ }^{a}$ Truscott Crisis Leaders, Wembley Downs, Western Australia 6019, Australia \\ Email: jtruscott@crisisleaders.com
}

\begin{abstract}
This CRISISLAB paper is aimed at managers who must simulate the leadership and coordination of their organization's response to any operational disruptive event or commercial hot issue. With CRISISLAB techniques it is possible to practice problem-solving in a simulated environment incorporating realtime high stress levels using shelfware, wetware and sweatware - crisis tools in effective Virtual Crisis Management Environments (VCME).

This paper provides an understanding of the actions needed to simulate the management of disasters in all forms. It describes the simulation of the visualization of stored and cross-related templates of our past experiences in VCME.

Through simulation, participants can then:

- determine how much effort should be put into crisis and business continuity preparation in their own business or improve their existing capability for high consequence risks,

- benchmark their own company's resilience under local and global levels at different times and different places,

- know how much documentation is enough (Shelfware Concepts),

- identify the competencies required of Executive Management Teams when confronted by risk issues (Wetware Capability),

- relate to the stress through having to devise strategy and plans and communicate under pressure in a CRISISLAB (Sweatware Technology),

- boost the volume and speed of message delivery, leverage multiple channels like phone, text and email, enable decision makers to work together, and track and respond to those who receive messages (Software as a Service VCME).
\end{abstract}

Keywords: CRISISLAB, crisis simulation, shelfware, wetware, sweatware 
Truscott, CRISISLAB - shelfware, wetware and sweatware: realization, actualization and simulation weapons of first resort

\section{BEST IN CLASS PRACTICES IN SIMULATIONS - DISASTER PREPARATION 101}

This paper is aimed at managers who must simulate the leadership and coordination of their organization's response to an operational event or commercial issue that becomes a crisis. The best organizations recognize that it is not enough to just have a plan. Crises are a turning-point, especially in relation to disease or a moment of danger or suspense in politics and commerce. It comes from the Greek word 'krino' to decide. Simulation gaming enables you to determine how much effort should be put into crisis and business continuity preparation in your organization and to improve your existing capability for high consequence risks. Technology benchmarking also enables you to determine your own organization's resilience at local and global levels and at different times and different places.

\section{MINDWORKS!}

How do you think big, think fast and think ahead in a simulation? High achievers such as elite athletes use affirmation and visualization where they state out loud or picture in their minds every day what it is they are trying to achieve to help fulfil their goals. The successful management and resolution of crises calls for people with answers, decisive strength, and a clear vision of the future, people who know where we ought to be going, people who can make hard problems seem simple. In short, leaders! In management teams we need a number of them. However in crises, instincts can be our undoing as studies on how we process information indicate that our wetware requires up to ten seconds to process bits of complex information. If you add more stress, coming from pressure of various types, the slower the process becomes. If we are not prepared for the possibility, when faced with multi-tasking our brains slow down just when we need to move much faster.

\section{DECISION MAKING UNDER STRESS}

Why do flight attendants shout orders to passengers repetitively? How do you simulate sitting in the hot seat? Perception-based retrieval of situationally appropriate responses is the dominant mode of responding but the additional need to integrate information and draw inferences places an even larger demand on working memory. This situation explains the urge to do nothing in a crisis. For example when people are asked to evacuate in anticipation of a natural disaster most people confer with several sources such as family, media and officials before making a decision. Such milling is common in disasters. People caught up in threatening circumstances fall into three categories. Ten to fifteen percent remain calm. Another fifteen percent breakdown although panic is usually isolated and controlled by the team around them. The vast majority of people remained stunned and bewildered. About forty-five percent of people shut down and stop moving or speaking for thirty seconds or often longer when asked under pressure to perform unfamiliar but basic tasks. They cease reasoning effectively and just sit there. It seems incredible that people are hard-wired to do nothing in a crisis. It is for this reason that people behave much more appropriately when they know what to expect (Ripley, 2005).

This is why crisis simulations provide organizations with a powerful means of selecting and training leaders and are an essential part of increasing any organization's resilience to business interruptions. Because of the potentially devastating effects the best companies constantly strive to successfully manage crisis situations. An important aspect in accomplishing this outcome is fostering leadership and a culture of deputies; the single-most critical factor in determining whether the crisis will be controlled. These crisis leaders have the crucial role of developing and implementing strategy under pressure with simulations providing a controlled and focussed environment in which to assess and train.

- Concepts $=$ Shelfware

- Capability = Wetware + Sweatware

- $\quad$ Technology = Virtual Crisis Management Environment (VCME)

Quite simply shelfware is just knowing how much documentation is enough; the bunker list approach and advance arrangements to event leadership. This is a concept and not all that useful in crises apart from the telephone directory. Wetware is being able to apply the competencies required of Executive Management Teams when confronted by risk issues. Sweatware is being able to endure the stress of a situation room or an operations room through having to devise strategy and plans and communicate under pressure. Wetware plus sweatware is capability. The CRISISLAB is a think tank putting it all together at different times and in different places. This is technology be it a game plan or web enabled. We will practice both shortly. 
Truscott, CRISISLAB - shelfware, wetware and sweatware: realization, actualization and simulation weapons of first resort

\section{CRISISLAB $=f(\underline{\text { Shelfware }+ \text { Wetware }+ \text { Sweatware }})$ \\ VCME}

When you put it all together it looks like this. When it comes together in a game plan these tools are realization and actualization and simulation weapons of first resort. In the field of disaster management and recovery people realize that some kind of preparation for a disaster is necessary. This is why a CRISISLAB is necessary.

Crisis simulations provide organizations with a powerful means of selecting and training its leaders to effectively handle crisis situations. Indeed the best method for training crisis leaders is the use of simulation. Military and sports teams, two groups that live under crisis-like stress, use simulations or scrimmage to develop the important instinct to act. The best time to discover a leader's crisis leadership capability is during a simulation and not during an actual crisis. It is an accepted axiom in the military and sport that people fight the way they train. The closer training simulates operations the better the person will act during an outage. Simulations are an effective means of predicting future job performance. This is done by providing stimuli that participants react to behaviourally and this behaviour can be observed (Yusko and Goldstein, 1997).

Accepting realism and feedback is a key to effective preparation for best crisis leadership. In developing the important instinct to act, it is accepted that people behave the way they prepare. So it is critical to practice problem-solving in a simulated environment incorporating real-time high stress levels underwriting the procedures leading towards resolution and beyond. Simulations with extensive realism and feedback are the keys to effective crisis leadership. Crisis leadership education must focus on how to prepare oneself and one's organization to anticipate and train for crises. Crisis leadership education also requires an understanding of how to prepare training, coaching, appropriate measurement and effective feedback mechanisms to improve the crisis leader (Elder). Crisis Management is like a sine wave. If there is too much action, there is a danger of acting too rashly. If there is too much planning, there is a danger of over analyzing and thus failing to act. It is all about balance between acting and analyzing. It is a unique Emergency Manager who can rapidly assimilate enough diverse information to make anticipatory decisions. Machines alone will not produce increased leadership ability without an accompanying increase in the Emergency Manager's ability to make decisions and issue orders.

\subsection{Analysis Versus Synthesis}

In simulations, crisis leaders must be drilled to ask themselves, what the worst possible thing is that could happen to my organization? The crisis leader must perform what if scenarios as to how they would handle the situation and how they would solve the problem in their organization. These scenarios can be informal, as simple as a mental exercise or visualization or very formal such as in a simulation. The crisis leader will identify groups and organizations that will help them to overcome problems for a crisis to one person maybe no crisis to another. Most people are not prepared for the crisis of being trapped in a burning building however to a fire fighter it is all in a day's work. How does the crisis leader know that they can perform under an actual crisis? The only way to know is to practice. Teams that specialize in crises use scenario practice to enable them to manage the stress of an actual crisis. Managers and emergency services all use war rooms or scenarios to hone and test their skills. Nearly all projects face setbacks, some severe enough to be considered a crisis. In many ways, handling a crisis is a project. Therefore crisis leaders should be trained like project managers to push past analysis paralysis, to give them the confidence to take charge and communicate in a convincing way to all stakeholders. This is accomplished by establishing the vision and communicating it. Effective thinking requires a balance between convergent thinking through which we seek to identify the best option and divergent thinking through which we seek to broaden our understanding of the problem and generate a wide range of options.

\subsection{Corporate Eskimo Roll - Business under Attack}

Hence you build confidence in people during a time of crisis by training and drilling before the crisis. Critical tasks and leadership procedures are drilled repeatedly until they become instinctive. The procedure is the process by which a leader establishes vision and communicates it. In business it is called the corporate Eskimo roll. The best plan never survives contact with a risk issue, but simulations can effectively raise issues, expose vulnerabilities, as well as lay the foundation for resolution. 
Truscott, CRISISLAB - shelfware, wetware and sweatware: realization, actualization and simulation weapons of first resort

- Emergenetics \& critical thinking, multitasking, prioritizing and delegating

- Remaining cool under pressure, making tough choices, empathizing

- Making quick and effective decisions

The fundamental job of the leader is to enforce strategy and to resist leadership compression; 'horizons not borders, solutions not differences, challenges not barriers.'

Leadership or its lack is often the most critical factor underwriting success or failure in managing a crisis. Success is more attitude than aptitude. So when do you act immediately and when do you delay your decision making? Perception-base retrieval of situationally appropriate responses is the dominant mode of responding to rule-based decisions. That means you act as you train. It is a drill. However choice decisions are more complex than rule-based decisions. The need to integrate information and draw inferences places a large demand on working memory, a process that is subject to stress effects and least amenable to a recognition-primed process. Creative problem-solving is needed when no response is readily available as a standard procedure, no guidance for handling a malfunction is to be found and when the team have not received training for the situation. Analogical processes maybe required in these situations (Flin et al., 1997).

\subsection{Mastering the Business Game of Snakes and Ladders - Decision making Under Stress}

No single theory of decision making can ever hope to fully capture the complexity of the mental processes engaged in by a decision maker faced with managing a crisis. However there are three ways to cope with uncertainty that blocks or delays action. You can actively collect additional information. You can passively defer decisions until additional information comes in, or you can fill in gaps through assumption-based reasoning. With these approaches you can continue to acknowledge uncertainty, you can ignore the uncertainty or you can deny any distorting knowledge, or you can generate alternatives. Such critical thinking is not always appropriate. Unless the risk of delay is acceptable, unless the cost of an error if one acts immediately is high and unless the situation is non-routine or problematic in some way the decision maker should probably act immediately. There is no doubt that critical thinking training can improve the accuracy of assessments. Recognition skills can be taught and that is the whole purpose of simulations (Flin et al., 1997). In the playground of the mind, imagination is the ultimate playground and immersion is the best introduction to this playground.

\subsection{Games without Frontiers}

Sanity is the playground for the unimaginative. Imagination is the ultimate playground. So how do you develop high quality, high fidelity crisis simulations for organizations? It is true that human beings will remember close to ninety percent of what they experience, as opposed to more like ten percent of what others tell them. Hence stressful simulations have a powerful, bonding effect on crisis teams when participants are empowered with personally owned experiences. The extreme leadership experience becomes embedded in the corporate knowledge base, and it enables organizations to simultaneously handle risks and seize potential opportunities. While there may be many lessons learnt from no notice events, simulations achieve maximum value if they are preceded by some form of refresher so that they are viewed by all as being successful, and the experience builds confidence in the organization's ability to regain control. Training also engenders management-in-depth, as deputies are often allocated responsibility in excess of their usual range. Simulations generally last less than half a day and are terminated when the crisis leader is implementing strategy. One controller observes the crisis team and the second controller directs the counter players. A counter players' room should be designated close to the crisis room, and each counter player must have access to a dedicated phone or a mobile phone. Crisis champions should conduct an immediate debrief of all participants to capture what they have learned from the exercise. This is a most valuable activity as the best plan never survives contact with a crisis. The aim is to share acquired corporate experience. Multi-layered simulations with a subordinate emergency dimension are best focused on the activation process and the management of information in crisis, as opposed to the end state of implementing successful business strategy. If this does not occur, multi-layered, top-down and bottom-up activities will generally become a compromise in value for one or both teams unless there are multiple counter players to simultaneously test both teams with credible scenario injects.

- Theatre of the Mind - Mind warfare and the battle of ideas in experimental rooms. Success is built on a mountain of failure. 
Truscott, CRISISLAB - shelfware, wetware and sweatware: realization, actualization and simulation weapons of first resort

In designing the crisis simulation, the purpose is to create stimuli that evoke the perception of a crisis and that allows a space within which the participants can react and provide behavioural responses. The goal is to create a realistic crisis situation that is representative of the type of organizational crisis that participants will have to handle (Yusko and Goldstein, 1997).

The crux of bringing about change is to provide a capstone event that allows participants to reflect on experience. To achieve change there needs to be a dislocation of expectations that allows movement in time.

\subsection{Cool under Fire - Shared Mental Models and Simulation Scenarios}

Scenarios should have commercial twists and turns so that they are more than operational events. For example they could pose continual moral dilemmas and the threat of the press and public opinion for each possible course of action. The scenarios should encompass known concerns, inevitable and unthinkable events, with and without an emergency dimension. Scenarios require strategy to be developed for business continuity, financial and legal liability and reputation issues. There may also be a strategic management of emergencies dimension requiring the endorsement of lower-order plans for saving life, protecting property and minimizing damage to the environment. There needs to be orchestrated confusion to force the participants down branches and sequels in its deliberations. Hence scenarios are prepared so that participants are forced to think about the future and not the past. Typical crises that come from cash, Industrial Relations, public perception, top management succession, sudden market shift, product failure, adverse international events, regulation and deregulation issues. What could cause the greatest grief for your organization; loss in client base or non-ethical reputation?

Some scenarios should have a non-operational flavour, such as an insidious governance issue or threat to director or officer of a company's fiduciary responsibilities. There should be no sub-sea valves to shut-in and the focus should be on handing back power to the Board. From this perspective Crises call for someone with answers, decision, strength, and a map of the future, someone who knows where we ought to be going - in short, someone who can make hard problems seem simple. Some scenarios may involve a double-jeopardy to pull the participants in two directions simultaneously. In these situations the dominant force should be a back-brief to the Chairman and the Board requiring participants to prioritize and think like businessmen rather than technocrats. The participants need to be pulled down by the weight of having to simultaneously strategically manage a major emergency. From this perspective Leaders must maintain their ability to keep thinking when a crisis develops and not become entangled in a swirl of anxiety that threatens to take over the system. Some scenarios should be fully virtual as is the predominant mode of corporate executives. This approach enforces team and time disciple in developing and implementing strategy under pressure. Participants need to experience true office rhythm. It may require selected individuals to speak publicly about the reputation of the organization in the court of public opinion and to apply downstream defensibility in the court of legal opinion. From this perspective Information is just knowledge. Experience is everything. Confidence is being able to apply that experience.

\subsection{Using Pre Stress to Avoid Distress}

The crisis situation should be realistic and engaging. The participants need to feel the heat. It cannot be a cool, rational, cognitive simulation. The emotions of being in a crisis must be activated. The consequences of the situation must be as real as possible so that participants experience stress. Actions and decisions should not be transparent. It cannot be so obvious that options become a single choice. Participants must be able to respond in many different ways with many different subsequent outcomes. There must be problems for each of the possible choices. Introducing new information, surprises and turns of events is a good way to achieve realism. These continual twists and turns over time are how actual crises often unfold. Background information is often necessary to pace the scenario in context particularly if there are to be limitations on resources to handle the crisis such as information, people and technology. There must be an allowance for effective participants to do well in a simulation (Yusko and Goldstein, 1997).

It is important to minimize no-win scenarios otherwise they are self defeating. While the usual aim for simulation is to build management capability and confidence, performance can be and often is measured across both personnel and procedures through peer and benchmark review, and against extant plans. Typically, a successful simulation is one in which the teams have demonstrated the ability to regain control and begin to plan effectively for the future. This will often come through the sometimes uncomfortable process of exposing vulnerabilities and deficiencies in accepted procedures and at times even in staff. Psychological and not just physical fidelity are 
Truscott, CRISISLAB - shelfware, wetware and sweatware: realization, actualization and simulation weapons of first resort

important. Simulations that focus on the physical can be expensive and so the focus is best on critical competencies (Yusko and Goldstein, 1997).

Having conducted hundreds of crisis simulations, many of them with some of the great companies in the world, we have found a main truth. Counter players are the engine room in simulation exercise activities. Actors and scenario injectors should be selected from deputies or alternates where possible and not Hollywood. Ideally they should be managers and staff who have a thorough understanding of the company's operations and business methods. So much so that companies with mature procedures will often exercise the alternates and utilize the primary members as counter players to power the exercise.

\section{CRISISLAB - SIMULATING BUSINESS UNDER ATTACK - PLAYGROUND OF THE MIND - IMMERSION TRAINING}

How do you do this? Firstly select a scenario as diverse as climate change, financial mismanagement or critical infrastructure breakdown. Use a Live Participants Brief to set out the game plan and it typically contains guidance on aim and objectives, start state and outline timings for the activity, participant's and counter player actions, controller's and observer's actions, resource limitations on desktop activities, passage of Information rules and the simulation contact directory. Use a counter player's brief for exercise planners and counter players eyes only. It contains an outline scenario as a story, detailed injects to be placed by counter players by telephone, email, fax or meeting and the list of stakeholders that are expected to be simulated. Locate the crisis team in the boardroom. Locate a counter player room close by and ensure a simple telephone and Internet system with the crisis team. Task these counter players under the direction of a controller to represent stakeholders such as media, shareholders and regulators. Initially brief the crisis team on the conduct of the exercise and the competencies being sought. Then conduct an intensive simulation for several hours which places the team under pressure to perform and requiring them to develop and implement business strategy under tight timelines. You can place any other number of assessors in the Boardroom. You can even invite potential employers to observe, sit in or even participate undeclared. You can eliminate some team members by calling them away or you can put more pressure on team members by nominating them as spokespersons for the media or injecting conflict that impacts on their role. Immediately debrief all participants.

- Coming out of Crisis is like wrestling a gorilla. You rest when the gorilla rests.

Hot wash-ups (Table 1) are a most valuable activity as the best plan never survives contact with a crisis and the aim is to share acquired experience and provide a growth enabling experience (Hermann, 1997). Here are typical key performance indicators for participants as crisis leaders. Apply the plan? Develop business strategy under pressure? Manage timeouts to regain control? Demonstrate crisis leadership and commercial teamwork? Communicate strategy to stakeholders? Use Support Teams to manage information flows? In short desktops without counter-play are just academic thinking. Like a hot bath they are forgotten about ten minutes later. Simulation success is an ability to apply correct psychology through practical application. This is the nexus.

Table 1. Knowledge, skills and attitudes.

\begin{tabular}{|c|c|c|}
\hline Knowledge & Skills & Attitudes \\
\hline
\end{tabular}

\subsection{Corporate Theatre - Tips for Great Captains of Chaos when Dealing with Weapons of Business Destruction - Simulation Master Class}

CRISISLAB is an intensive simulation which places the team under pressure to perform and which requires them to develop and implement business strategy under tight timelines. It provides participants with an understanding of the strategies and executive actions needed to manage disasters, crises, emergencies and business outages. Participants learn how to simulate overcoming the Amygdala Hijack; when the brain's emotional memory bank 
Truscott, CRISISLAB - shelfware, wetware and sweatware: realization, actualization and simulation weapons of first resort

freezes. In these fast moving sessions participants work as industry aligned Management Teams to develop and implement business continuity, liability and reputation strategy and plans under pressure for a scenario which require simultaneous intervention by their Crisis Management Team, Emergency Management Team and Emergency Services. Participants step through a scenario requiring layered Management Teams to find and check the facts, identify stakeholders and prioritize issues, brainstorm potential strategies and develop a path way to the future. The best Management Teams are multi-tasking, using plenary sessions and timeouts to achieve team and time discipline. All participants are challenged to interact. Several participants may be asked to provide updates as CEO and spokespersons while formulating and communicating strategy. Others participants maybe asked questions in a post crisis witness box.

- The darkest corner in hell is reserved for fence-sitters (Dante).

All executives emerge with cohesion when they participate in these extreme leadership sessions and prove that they can align their communications with the operational response and achieve the objective of developing and implementing strategy under pressure before returning to Line Management. In coming out of crisis where to from here? Scenarios are designed to raise issues as much to solve them. As explorers of business continuity, to practice crisis leaders you must be given problems that you have not solved before.

\subsection{Using Simulations to Identify Managers with a Determination to Lead}

Real leaders might have saved Enron as an institution takes on the character of its leadership. As anxiety is contagious, so is serenity. Panic is contagious but so is courage. What competencies are you after? Can the person do the job? Will the person do the job? Will the person fit into the organization? Competencies are more valuable than skills and corporate searches for leaders are becoming harder. Managers are not paid for the routine work they do today, but rather for what they might have to do in the extraordinary circumstance that is tomorrow. The art of crisis leadership is thus the ability not only to contain the problem when it occurs but to take the organization past any advantage line and transform the organisation post-crisis. Hence crisis leaders are bigpicture, people types who use power and passion to turn the camera on in people's minds. Leaders must maintain their ability to think clearly during an emergent crisis rather than becoming swept up in the panic of crisis hysteria. Theirs is the crucial task of developing a strategy aimed at resolution, and of articulating it to their organisation through raw displays of leadership in the workplace. Simulations are being successfully used to predict future job performance, determine managerial potential and to gather information for promotion. High performance evolutions have been consistently linked to the bottom-line effectiveness of organizations who create high-fidelity, realistic scenarios that mimic the characteristics of a crisis (Yusko and Goldstein, 1997).

\section{REFERENCES}

Elder, A.L. Developing Crisis Leadership Competencies. Numen Consulting Inc.

Flin, R., Salas, E., Strub, M., and Marton, L. eds. (1997). Decision Making Under Stress, Emerging Themes and Applications. Ashgate Publishing, 1997.

Hermann , M.G. (1997). In Conclusion: The Multiple Pay-Offs of Crisis Simulations. Journal of Contingencies and Crisis Management, Volume 5, Number 4, December 1997.

Ripley, A. (2005). How to Get Out Alive, From Hurricanes to 9/11: What the Science of Evacuation Reveals about How Humans Behave in Disasters. 2 May 2005.

Yusko, K.P., and Goldstein, H.W. (1997). Selecting and Developing Crisis Leaders Using Competency-Based Simulations. Journal of Contingencies and Crisis Management, Volume 5, Number 4, December 1997. 